\title{
Venous thromboembolism in patients with inflammatory bowel disease : review of literature and practical algorithms
}

\author{
L. Coremans ${ }^{1}$, B. Strubbe ${ }^{2}$, H. Peeters ${ }^{2}$ \\ (1) Ghent University and AZ Sint-Lucas, Ghent, Belgium ; (2) Department of Hepatology and Gastroenterology, AZ Sint-Lucas, Ghent, Belgium.
}

Table 1.

\begin{abstract}
Inflammatory bowel disease (IBD) is associated with several extra-intestinal complications, including venous thromboembolism (VTE). In patients with IBD, VTE occurs at younger age and is associated with higher recurrence and mortality rates as compared to patients without IBD. The risk appears to be higher during active disease and hospitalization.

In this review we target the importance of prophylaxis and aim to describe strategies for treatment of VTE in patients with IBD. More awareness is needed, given the fact that VTE is often preventable with appropriate pharmacological prophylaxis. Algorithms are provided on which patients should be given prophylaxis and on treatment duration of VTE in patients with inflammatory bowel disease. (Acta gastroenterol. belg., 2021, 84, 79-85).
\end{abstract}

Keywords : Inflammatory bowel disease, Crohn's disease, ulcerative colitis, venous thromboembolism

\section{Introduction}

Venous thromboembolism (VTE) is considered one of the most common extra-intestinal complications of inflammatory bowel disease (IBD). Several large population-based cohort studies and meta-analyses have demonstrated a two- to three-fold increased risk of VTE in patients with IBD compared to the general population (1-3). In a nationwide multicenter study in Austria of 2013 including 2811 patients with IBD, the prevalence and incidence rate of all VTE was $5.6 \%$ and 6.3 per 1000 person-years, respectively (4). A large UK cohort study of 2010 demonstrated an absolute risk of $2 \cdot 6$ per 1000 person-years for patients with IBD as compared to the general population, with a hazard ratio of 3.4 (3). The risk appears to be similar for Crohn's disease (CD) as for ulcerative colitis (UC) but increases with active disease and hospitalization (3).

In the eight edition (2008) of the American College of Chest Physician (ACCP) guidelines on prevention of VTE, IBD was included as a risk factor (5). However, in the subsequent and most recent edition of 2012, IBD is no longer included (table 1) (6).

IBD seems to be a disease-specific risk factor for VTE. This is supported by the finding that other chronic inflammatory diseases, such as rheumatoid arthritis, or chronic bowel diseases, such as celiac disease, do not seem to have an increased risk of VTE (7).

In this review we target the importance of prophylaxis and aim to describe strategies for treatment of VTE in patients with IBD.

\begin{tabular}{|l|l|}
\hline $\begin{array}{l}\text { Geerts et al, ACCP guidelines 8th } \\
\text { edition (2008) }\end{array}$ & $\begin{array}{l}\text { Kahn et al, ACCP guidelines } \\
\text { 9th edition (2012) }\end{array}$ \\
\hline Cancer, active or occult & Active cancer \\
\hline $\begin{array}{l}\text { Cancer therapy (hormonal, chemo- } \\
\text { therapy, radiotherapy, angiogenesis } \\
\text { inhibitors) }\end{array}$ & $/$ \\
\hline Previous VTE & $\begin{array}{l}\text { Previous VTE (with the ex- } \\
\text { clusion of superficial vein } \\
\text { thrombosis) }\end{array}$ \\
\hline Immobilization > 3 days & Immobilization > 3 days \\
\hline Inherited or acquired thrombophilia & $\begin{array}{l}\text { Known thrombophilic condi- } \\
\text { tion }\end{array}$ \\
\hline Recent trauma/surgery & $\begin{array}{l}\text { Recent (< 1mo) trauma/ } \\
\text { surgery }\end{array}$ \\
\hline $\begin{array}{l}\text { Venous compression (tumor, hema- } \\
\text { toma, arterial abnormality) }\end{array}$ & $/$ \\
\hline$/$ & Cardiac/respiratory failure \\
\hline$/$ & $\begin{array}{l}\text { Recent myocardial infarction } \\
\text { or ischemic stroke }\end{array}$ \\
\hline / & $\begin{array}{l}\text { Acute infection and/or rheu- } \\
\text { matologic disorder }\end{array}$ \\
\hline Increasing age & Age $>$ 70 years \\
\hline Obesity & Obesity (BMI > 30) \\
\hline $\begin{array}{l}\text { Oral contraceptive, ongoing hor- } \\
\text { monal treatment }\end{array}$ & $\begin{array}{l}\text { Oral contraceptive, ongoing } \\
\text { hormonal treatment }\end{array}$ \\
\hline Pregnancy and post-partum period & $/$ \\
\hline Erythropoiesis-stimulating agents & $/$ \\
\hline Acute medical illness & $/$ \\
\hline Inflammatory bowel disease & $/$ \\
\hline Nephrotic syndrome & $/$ \\
\hline Myeloproliferative disorders & $/$ \\
\hline $\begin{array}{l}\text { Paroxysmal nocturnal hemoglobi- } \\
\text { nuria }\end{array}$ & $/$ \\
\hline Centrale venous catherization & $/$ \\
\hline & \\
\hline
\end{tabular}

Venous thromboembolic complications in IBD

Most commonly deep vein thrombosis (DVT) and pulmonary embolism (PE) are observed (8). Numerous other less frequent sites of venous thrombosis such as mesenteric and portal vein thrombosis and retinal vein occlusion as well as arterial thrombosis have been described in patients with IBD (9).

Correspondence to : Coremans Laura, Ghent University Hospital/ AZ Sint-Lucas Ziekenhuis, Groene Briel 1, 9000 Ghent, Belgium. Phone : 0032473672967. Fax : 003292246488.

E-mail : laura.coremans@ugent.be

Submission date : 27/06/2020

Acceptance date : $13 / 10 / 2020$ 
VTE occurs earlier in life in patients with IBD as compared to non-IBD patients and has a higher recurrence rate (10). In a large, nationwide Danish study in 2011 the incidence of VTE increased with age as expected, however the relative risk for occurrence of unprovoked VTE (not triggered by trauma, surgery, or pregnancy) was higher at young ages. In IBD patients aged 20 years or younger the hazard ratio for unprovoked VTE was 4.5 (confidence interval 1.7 to 12.0 ) as opposed to 1.4 (confidence interval 1.2 to 1.6 ) in IBD patients 60 years or older (2). When compared to non-IBD patients presenting with VTE, the incidence rates at 20 years or younger was markedly higher in patients with IBD ( 8.9 per 10000 person-years versus 2.0 per 10000 personyears in non-IBD patients, p-value not provided) (2). There were no significant differences in incidence rates between gender and disease type (UC versus CD) $(2,10)$.

In a cohort study (2010) comparing 86 patients with IBD to 1255 patients without IBD, all with a first unprovoked episode of VTE, the recurrence rate 5 years after discontinuation of anticoagulation therapy was significantly higher in patients with IBD (33.4\% probability of recurrence versus $21.7 \%, \mathrm{p}=0.01$ ) (11). Younger age at first episode (median age 43.9 , IQR 35.6-56.2) is also correlated with a significant increased risk of recurrence (hazard ratio $1.03 ; \mathrm{p}=0.03$ ).

In patients with IBD the absolute risk of VTE is most elevated during active disease (defined as corticosteroid use) and during hospitalization. In a large UK cohort study published in 2010 including 13.756 patients with IBD and 71.672 matched controls, Grainge et al demonstrated a significantly increased overall risk of VTE in IBD patients compared to controls during all activity phases (acute flare, chronic activity and remission), after adjusting for age, sex, cardiovascular risk factors and history of PE or DVT (3). Incidence rates increase during hospitalized periods $(17 \cdot 8$ per 1000 person-years as opposed to $0 \cdot 6$ per 1000 person-years during ambulatory periods).

Furthermore the extent of the disease also correlates with a higher risk of VTE (12). In a retrospective study of 2004 evaluating clinical features of IBD patients with VTE, pancolonic involvement was seen in $76 \%$ of patients with UC $(\mathrm{n}=59)$ and ileocolonic involvement in $56 \%$ of patients with $\mathrm{CD}(\mathrm{n}=39)$ (13). Other risk factors include colonic involvement for $\mathrm{CD}$, complicated disease (fistulas, stenosis, abscesses) and recent surgery.

However a specific risk factor for VTE is not always present, supporting the hypothesis that IBD is an independent risk factor for VTE (14). In the study by Grainge et al up to $30 \%$ of VTEs occurred when the disease was in remission (3).

\section{Tofacitinib and increased risk for VTE}

Tofacitinib, an oral small-molecule JAK- inhibitor has been approved for the treatment of moderately to severely active UC after proving efficacy as induction and maintenance therapy in 3 large clinical trials (15). In February 2019 the Food and Drug Administration (FDA) released a black box warning on the potentially increased risk for VTE in patients with rheumatoid arthritis (RA) treated with high dose Tofacitinib (e.g. 10mg twice daily) (16). This was based on data from the ORAL surveillance study, an ongoing clinical trial evaluating the risk of major cardiovascular events (MACE) in RA patients older than 50 years and with at least one cardiovascular risk factor during treatment with Tofacitinib as compared to treatment with tumor necrosis factor (TNF)a antagonists (17). In an interim analysis, a 5-fold increased incidence for $\mathrm{PE}$ and higher all-cause mortality was found in patients treated high dose Tofacitinib as opposed to (TNF) a antagonists. Subsequently a large observational cohort study in 2019 found numerically higher, but statistically nonsignificant risk for VTE in RA patients receiving Tofacitinib versus those receiving (TNF)a antagonists (IR per 100 person-years $0.77,95 \%$ CI of $0.43-1.27$, per 100 person- years) (18).

In the OCTAVE open study, an ongoing, open-label, long-term extension study including 1157 UC patients, 1 patient developed DVT (IR $0.04 ; 0.00-0.23$ ) and 4 patients developed PE (IR 0.16;0.04-0.41) (19). All patients received maintenance high dose Tofacitinib but all patients had other risk factors for VTE (cancer, prior history of VTE, recent immobilization or recently started CHC). In a retrospective study of 2019 evaluating adverse events in $260 \mathrm{UC}$ patients receiving treatment with Tofacitinib during median follow-up time of 6 months, 2 patients $(0.8 \%)$ developed VTE (20). Both patients were receiving high dose $10 \mathrm{mg}$ twice daily and had extensive disease. In July 2019 the FDA released an updated safety announcement limiting the use of high dose Tofacitinib in UC patients to initial induction therapy (21).

Beyond 8 weeks of therapy high dose Tofacitinib should only be used in limited situations after a careful evaluation of risks and benefits, especially in patients with pre-existing risk factors for VTE (20).

\section{Pathogenesis}

Inflammatory bowel disease seems to be a diseasespecific risk factor for VTE, but the exact pathogenesis for this increased risk is unknown. The endothelium seems to play a crucial role in the hypercoagulable state, due to both intestinal and systemic inflammation (14). This is known as the vascular hypothesis. Chronic intestinal inflammation generates oxidative stress, endothelial dysfunction and multifocal intestinal vascular infarcts. Several prothrombotic abnormalities have been described, such as initiation of the coagulation system, impairment of fibrinolysis and induction of platelet abnormalities, with increased activation and aggregation (22). Intestinal inflammation is more pronounced during active disease, consequently this could explain the higher incidence of VTE during active disease.

Patients with IBD seem to have an altered clot lysis profile, possibly explaining the increased risk for VTE 
(23). Therefore, analysis of this clot lysis profile could be included in the thromboembolic risk assessment and used as specific biomarker. Specifically hypofibrinolysis and increased concentrations of fibrinolysis inhibitors, such as active plasminogen activator inhibitor 1 (PAI1) and activated thrombin activatable fibrinolysis inhibitor (TAFIa) seem to be involved in the increased thromboembolic risk in IBD patients (24). In 2015, a case-control study comparing the clot lysis profile in IBD patients with and without prior VTE versus healthy controls showed that increased concentrations of fibrinolysis parameters are significantly associated with the presence of IBD in general (24). In IBD patients with prior VTE, clot lysis parameters were significantly higher when compared to IBD patients without prior history of VTE.

Because active inflammation is associated with increased thromboembolic risk, effective antiinflammatory therapies could influence the hemostatic profile and subsequently reduce the thromboembolic risk (25). A prospective study of 2015 compared clot lysis profile in 103 IBD patients before and after initiating of Infliximab with healthy controls (25). In primary responders after induction therapy (week 14), a decrease in inflammatory markers was associated with normalization of the clot lysis profile, reflected by significantly decreased concentrations of fibrinolysis inhibitors $(\mathrm{p}=0.01)$.

Inherited thrombophilia such as factor $\mathrm{V}$ Leiden, protein $\mathrm{C} / \mathrm{S}$ deficiency and antithrombin deficiency occur in a similar frequency in patients with IBD as compared to non-IBD patients, therefore genetics do not seem to explain the increased risk of VTE (26).

\section{Prevention of thromboembolism in IBD patients}

In comparison to the general population, VTE in patients with IBD seems to be associated with worse prognosis with longer hospital admissions and higher mortality rates. In a nation-wide study in the USA (2008) age- and comorbidity-adjusted excess mortality from VTE was 2.1-fold higher for patients with IBD as compared to non-IBD patients $(p<0.01)(27)$. Inhospital mortality for IBD patients presenting with VTE was significantly higher when compared to IBD patients without VTE, both for CD (17.0 vs 4.2 per 1000 hospitalizations, $\mathrm{p}<0.01)$ and UC (37.4 vs 9.9 per 1000 hospitalizations, $\mathrm{p}<0.01$ ).

Among hospitalized patients with IBD, the average length of stay was almost twice as long in patients with VTE compared to those without VTE (11.7 days vs 6.1 days, $\mathrm{p}<0.01)(27)$.

The European ECCO guideline on extra-intestinal complications in IBD (2016) state that the risk for VTE is related to IBD activity and prophylaxis is recommended for all hospitalized patients with IBD (28). Furthermore consideration of VTE prophylaxis is recommended after discharge from hospital, after recent surgery and in ambulatory patients with active disease. However, more details on patient selection and duration of thromboprophylaxis are limited. Except for the consensus statement of the Canadian association of Gastroenterology there are no other clear international guidelines for the prevention or treatment of VTE in patients with IBD (29).

Prevention of VTE is possible and involves both mechanical and pharmacological prophylaxis. For each IBD patient presenting with VTE, an assessment of individual risk factors for VTE and consequently, correction of risk factors that are modifiable should be made (e.g. obesity, oral contraceptives, smoking) (30).

\section{Hospitalized patients}

Pharmacological thromboprophylaxis is recommended in all hospitalized patients with IBD, regardless of the reason for admission. (GRADE strong recommendation, low-quality evidence) (29).

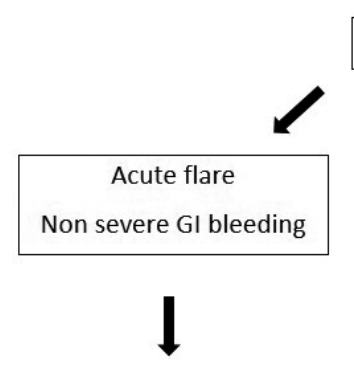

Prophylaxis with LMWH

Or unfractionated heparin

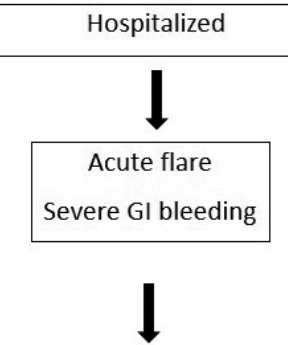

Mechanical prophylaxis

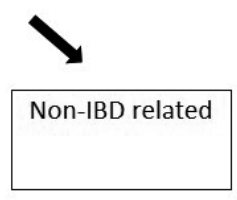

Prophylaxis with LMWH

Or unfractionated heparin

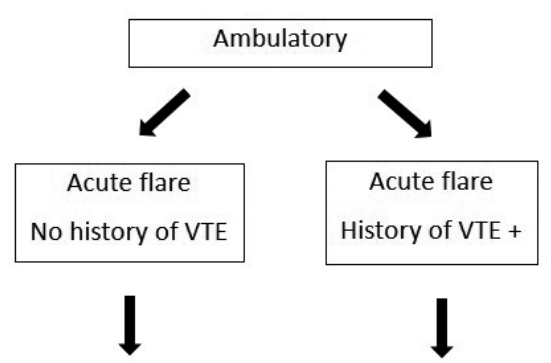

No prophylaxis

Prophylaxis with LMWH
GRADE

weak recommendation Very low-quality evidence

Figure 1. - Suggested algorithm for primary prophylaxis in hospitalized and ambulatory patients. Adapted from : Nguyen et al. Consensus statements on the risk, prevention, and treatment of venous thromboembolism in inflammatory bowel disease : Canadian Association of Gastroenterology. Gastroenterology, 2014. Abbreviations : GI : gastro-intestinal. LMWH : Low-molecular-weight heparin. IBD : inflammatory bowel disease. VTE : venous thromboembolism. 
Only in patients with severe (gastro-intestinal) bleeding who are hemodynamically instable, mechanical thromboprophylaxis is recommended. After hemodynamic stability is obtained pharmacological prophylaxis should be initiated. (GRADE weak recommendation, low-quality evidence) (29).

Hospitalization is an important risk factor for VTE in patients with IBD, for patients with active disease as well for patients in remission. It is therefore recommended that all IBD patients hospitalized for an acute flare should be given prophylaxis (figure 1) (28,29). More specifically, hospitalized patients with acute severe colitis, despite blood loss, have the highest risk for VTE (3). Therefore pharmacological prophylaxis is exceedingly important in these patients. Most commonly low-molecular-weight heparin (LMWH) are used, but low-dose unfractionated heparin is also an option.

Only in patients with severe (gastro-intestinal) bleeding, defined as patients who are hemodynamically compromised, pharmacological prophylaxis should be withheld. In this case, mechanical thromboprophylaxis is recommended, preferably intermittent pneumatic compression (IPC) (29). As soon as the bleeding is controlled and hemodynamic stability is obtained, switching to pharmacological prophylaxis is recommended.

In patients who are hospitalized for reasons other than IBD, including those in clinical remission, primary prophylaxis is also recommended. This is derived from data from the large UK cohort study indicating that the absolute risk of VTE in hospitalized IBD patients in remission is 3 -fold higher than in nonhospitalized patients with an acute flare (3). Moreover, pharmacological prophylaxis seems to be safe in IBD patients. In a large retrospective study of 2013, there was no difference in occurrence of major or minor bleeding between hospitalized IBD patients who did and did not receive pharmacological prophylaxis (31).

\section{Ambulatory patients}

Pharmacological thromboprophylaxis in ambulatory patient is only recommended in case of an acute flare if there is a history of VTE. (GRADE weak recommendation, very low-quality evidence) (29).

In ambulatory patients presenting with an acute flare the decision to give pharmacological prophylaxis depends on prior history of VTE. In outpatients without previous VTE, pharmacological therapy is not recommended (figure 1) (29). Outpatient flares of IBD are associated with a 16-fold increased risk of thrombosis, but the absolute risk is low ( $0.16 \%$ during a 3 month period) (3). However, in ambulatory IBD patients with a history of previous VTE pharmacological prophylaxis is suggested. This is derived from the assumption that in patients with a history of VTE, an IBD flare is considered a provoking but reversible risk factor.

Specific concerns exist on the use of combined hormonal contraception ( $\mathrm{CHC}$, combining both an estrogen and a progestogen) in female IBD patients and the possible accumulating risk of VTE. The U.S. Medical Eligibility Criteria for Contraceptive Use (2016) state that in female IBD patients with increased risk for VTE (active or extensive disease, surgery, immobilization, corticosteroid use) the risk of $\mathrm{CHC}$ use generally outweighs the benefits (32). However, in women with mild IBD and with no other risk factor for VTE the benefits of CHC use generally outweigh the risks (32). Moreover, a retrospective study of 2015 found no increased risk of VTE in female IBD patients in remission receiving oral contraceptives when compared to non-IBD patients receiving oral hormonal contraceptives (33).

\section{Treatment and duration of anticoagulation therapy for acute VTE}

The European ECCO guideline on extra-intestinal manifestations in IBD states that the same treatment protocols can be used for VTE in patients with IBD as for non-IBD patients (28). Most commonly vitamin K antagonists or low-molecular-weight heparin (LMWH) are used. There is insufficient data on the use of direct oral anticoagulants (DOAC) in patients with IBD and VTE. In a study of 2018 based on data from the RIETE registry, an ongoing international observational registry of patients with VTE, only 2 out of 180 patients with VTE and IBD in remission were treated with DOAC (Rivaroxaban) (34). Data from the general population suggest that Apixaban is associated with less non-major bleeding episodes than both Rivaroxaban or Dabigatran $(28,35)$.

In general, the efficacy and safety of anticoagulation therapy seems the be the same for both IBD and non-IBD patients. There was no statistically significant difference in the rate of VTE recurrence nor in major bleeding in IBD patients with acute flare, IBD patients in remission and non-IBD patients (34).

\section{Acute flare}

If a risk factor can be identified in IBD patients presenting with acute VTE, anticoagulation therapy should be continued for 1 month after resolution of the risk factor. (GRADE strong recommendation, lowquality evidence) (29)In patients with an acute flare but no other risk factor, anticoagulation therapy should be continued for 3 to 6 months after remission. (GRADE strong recommendation, very low-quality evidence) (29).

In IBD patients with active disease presenting with a first episode of VTE, anticoagulation therapy is recommended for 3 months after remission is achieved if no other risk factor can be identified (figure 2). This is based on the assumption that active disease is a reversible, provoking risk factor (36).

Alternatively, if another independent risk factor can be identified, anticoagulation therapy should be continued for 1 month after resolving the risk factor and for at 


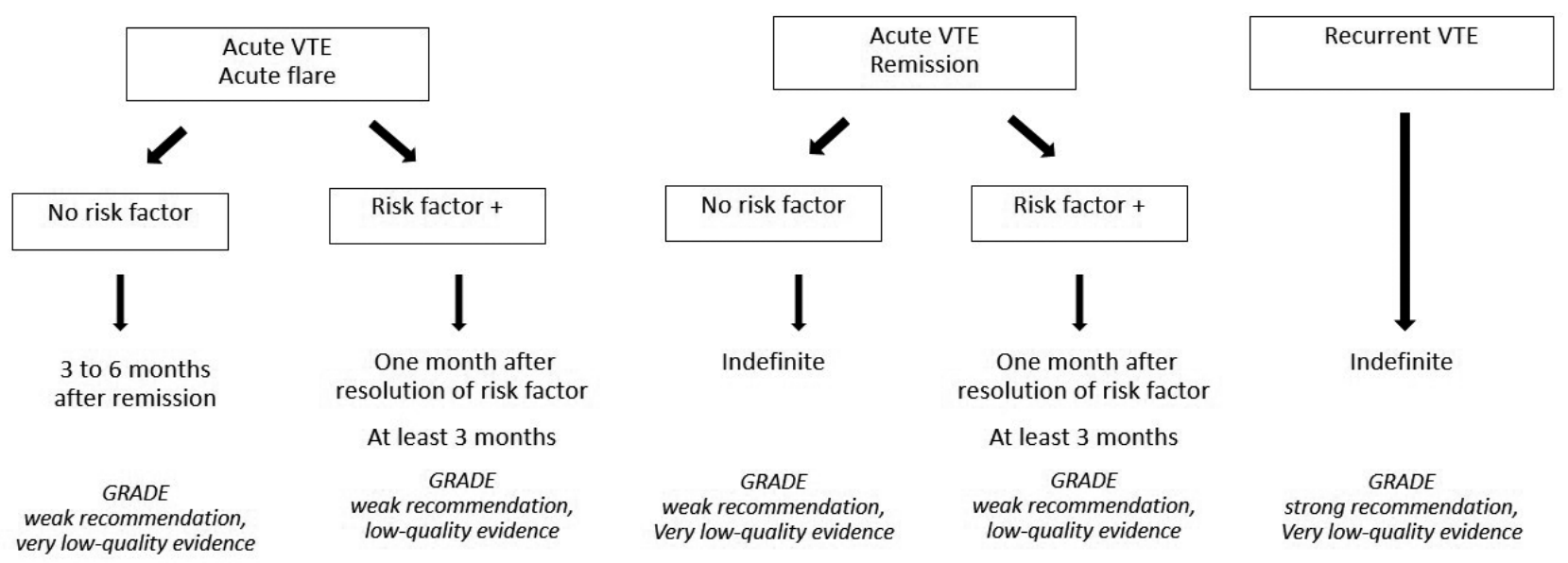

Figure 2. - Suggested algorithm for treatment duration of VTE during different activity phases. Adapted from : Nguyen et al. Consensus statements on the risk, prevention, and treatment of venous thromboembolism in inflammatory bowel disease : Canadian Association of Gastroenterology. Gastroenterology, 2014. Abbreviations : VTE : venous thromboembolism.

least 3 months (37). Patients with VTE presenting with an identifiable risk factor have a much lower risk of recurrence (36). Consequently in female IBD patients with an acute flare and taking combined hormonal contraception when presenting with a first episode of VTE, stopping hormonal contraception should be considered (32).

\section{Remission}

For IBD patients presenting with acute VTE while in remission and in the absence of another risk factor, indefinite anticoagulation therapy is recommended. (GRADE strong recommendation, very low-quality evidence) (29).

Patients with IBD who are in clinical remission when diagnosed with a first episode of VTE and in the absence of another provoking factor, indefinite anticoagulation therapy is suggested in the ECCO guideline (figure 2) (28).

In a cohort study, in 86 out of 166 patients with a first episode of VTE a risk factor could not be identified and were therefore considered "unprovoked". In these patients, the 5-year risk of recurrence was estimated at $33 \%$ after discontinuation of anticoagulation therapy (11). Therefore in the Canadian consensus statement, in IBD patients without an additional provoking risk factor, IBD itself was considered an ongoing risk factor for recurrent VTE (38).

In IBD patients with recurrent VTE ( 2 or more events) indefinite anticoagulation therapy with LMWH of vitamin $\mathrm{K}$ antagonists is also recommended (figure 2).

\section{Conclusions}

Inflammatory bowel disease is associated with a higher risk of VTE, especially during acute flares and hospitalized periods. In patients with IBD, VTE occurs at younger age and has higher recurrence and mortality rates. Therefore more clinical awareness is needed. Thromboembolism is preventable by the use of appropriate pharmacological prophylaxis. Individual assessment of risk factors for VTE and, if possible, elimination of modifiable risk factors should be attempted, especially during acute flares.

Algorithms which can be used in clinical practice are provided on which patients should be given prophylaxis and duration of treatment of acute VTE in IBD patients.

Pharmacological prophylaxis is recommended in all hospitalized patients with IBD, regardless of disease activity. Especially in hospitalized patients with acute severe colitis, the risk for VTE is very high and pharmacological prophylaxis is recommended, despite (gastro-intestinal) blood loss. Only in patients with severe (gastro-intestinal) bleeding, defined as hemodynamically unstable, pharmacological prophylaxis should be withheld and replaced by mechanical thromboprophylaxis. However as soon as the bleeding is controlled and hemodynamic stability is regained, switching to pharmacological prophylaxis is recommended.

In ambulatory IBD patients with an acute flare, pharmacological prophylaxis should only be initiated if there is a prior history of VTE, given the low absolute risk of VTE.

In patients with an acute flare presenting with VTE, the duration of anticoagulation treatment is based on the presence of another risk factor. In the presence of another risk factor, therapy should be continued for 1 month after resolution of the risk factor and for at least 3 months. If not, anticoagulation therapy is recommended for 3 months after remission.

For patients diagnosed with VTE while in clinical remission, duration of treatment also depends on the presence of another risk factor. If a risk factor cannot be identified, indefinite anticoagulation therapy is recommended, assuming IBD itself is an ongoing risk factor for recurrent VTE. 
Before initiating treatment with Tofacitinib in UC patients, evaluation for other thromboembolic risk factors is warranted. Use of high dose Tofacitinib beyond the 8 weeks induction phase should only be used in limited situations. Further studies are needed to characterize the association between treatment with Tofacitinib and VTE in specific patient populations.

Further questions to be addressed are the need to discontinue combined hormonal contraception in female IBD patients with concomitant risk factors for VTE, the efficacy and safety of DOAC in the treatment of VTE in patients with IBD and potential need and the duration of pharmacological prophylaxis during the posthospitalization period.

\author{
Abbreviations \\ $\mathrm{CD}:$ Crohn's disease \\ CHC : Combined hormonal contraception \\ DOAC : direct oral anticoagulant \\ DVT : deep vein thrombosis \\ IBD : inflammatory bowel disease \\ LMWH : Low-molecular-weight heparin \\ PAI-1 : active plasminogen activator inhibitor 1 \\ PE : pulmonary embolism \\ RA : rheumatoid arthritis \\ TAFIa : activated thrombin activatable fibrinolysis inhibitor \\ TNF : tumor necrosis factor \\ UC : ulcerative colitis \\ VTE : venous thromboembolism
}

\section{Conflict of interest}

None. The authors declare that they have no competing interests.

\section{Funding}

No funding was provided.

\section{References}

1. YUHARA H, STEINMAUS C, CORLEY D, KOIKE J, IGARASHI M, SUZUKI T, et al. Meta-analysis : the risk of venous thromboembolism in patients with inflammatory bowel disease. Aliment. Pharmacol. Ther., 2013, 37(10) : 953-62.

2. KAPPELMAN MD, HORVATH-PUHO E, SANDLER RS, RUBIN DT, ULLMAN TA, PEDERSEN L, et al. Thromboembolic risk among Danish children and adults with inflammatory bowel diseases : a population-based nationwide study. Gut, 2011, 60(7) : 937-43.

3. GRAINGE MJ, WEST J, CARD TR. Venous thromboembolism during active disease and remission in inflammatory bowel disease : a cohort study. Lancet, 2010, 375(9715) : 657-63.

4. PAPAY P, MIEHSLER W, TILG H, PETRITSCH W, REINISCH W, MAYER $\mathrm{A}$, et al. Clinical presentation of venous thromboembolism in inflammatory bowel disease. J. Crohns Colitis, 2013, 7(9) : 723-9.

5. GEERTS WH, BERGQVIST D, PINEO GF, HEIT JA, SAMAMA CM, LASSEN MR, et al. Prevention of venous thromboembolism: American College of Chest Physicians Evidence-Based Clinical Practice Guidelines (8th Edition). Chest, 2008, 133(6 Suppl) : 381s-453s.

6. KAHN SR, LIM W, DUNN AS, CUSHMAN M, DENTALI F, AKL EA, et al. Prevention of VTE in nonsurgical patients : Antithrombotic Therapy and Prevention of Thrombosis, 9th ed : American College of Chest Physicians Evidence-Based Clinical Practice Guidelines. Chest, 2012, 141(2 Suppl) : e195S-e226S.
7. MIEHSLER W, REINISCH W, VALIC E, OSTERODE W, TILLINGER $\mathrm{W}$, FEICHTENSCHLAGER $\mathrm{T}$, et al. Is inflammatory bowel disease an independent and disease specific risk factor for thromboembolism? Gut, 2004, 53(4) : 542-8.

8. ZITOMERSKY NL, VERHAVE M, TRENOR CC, 3RD. Thrombosis and inflammatory bowel disease : a call for improved awareness and prevention. Inflamm. Bowel Dis., 2011, 17(1) : 458-70.

9. MARASCO G, SCAIOLI E, ZAGARI RM, BELLUZZI A. Aortic thrombosis recurrence in a Crohn's disease patient. Acta Gastroenterol. Belg., 2018, 81(4) : 546.

10. BERNSTEIN CN, BLANCHARD JF, HOUSTON DS, WAJDA A. The incidence of deep venous thrombosis and pulmonary embolism among patients with inflammatory bowel disease : a population-based cohort study. Thromb. Haemost., 2001, 85(3) : 430-4.

11. NOVACEK G, WELTERMANN A, SOBALA A, TILG H, PETRITSCH W, REINISCH W, et al. Inflammatory bowel disease is a risk factor for recurrent venous thromboembolism. Gastroenterology, 2010, 139(3) : 779-87, 87.e1.

12. TALBOT RW, HEPPELL J, DOZOIS RR, BEART RW, JR. Vascular complications of inflammatory bowel disease. Mayo Clin. Proc., 1986, 61(2) : $140-5$.

13. SOLEM CA, LOFTUS EV, TREMAINE WJ, SANDBORN WJ. Venous thromboembolism in inflammatory bowel disease. Am. J. Gastroenterol., 2004, 99(1) : 97-101

14. ZEZOS P, KOUKLAKIS G, SAIBIL F. Inflammatory bowel disease and thromboembolism. World J. Gastroenterol., 2014, 20(38) : 13863-78.

15. SANDBORN WJ, SU C, SANDS BE, D'HAENS GR, VERMEIRE S, SCHREIBER S, et al. Tofacitinib as Induction and Maintenance Therapy for Ulcerative Colitis. N. Engl. J. Med., 2017, 376(18) : 1723-36.

16. US FDA. Safety trial finds risk of blood clots in the lungs and death with higher dose of tofacitinib (Xeljanz, Xeljanz XR) in rheumatoid arthritis patients ; FDA to investigate February 25, 2019. Available from : https:// www.fda.gov/drugs/drug-safety-and-availability/safety-trial-finds-riskblood-clots-lungs-and-death-higher-dose-tofacitinib-xeljanz-xeljanz-xr.

17. ClinicalTrials.gov. Safety Study Of Tofacitinib Versus Tumor Necrosis Factor (TNF) Inhibitor In Subjects With Rheumatoid Arthritis. Identifier : NCT02092467 [Internet]. March 2014. Available from : https://clinicaltrials. gov/ct2/show/NCT02092467.

18. DESAI RJ, PAWAR A, WEINBLATT ME, KIM SC. Comparative Risk of Venous Thromboembolism in Rheumatoid Arthritis Patients Receiving Tofacitinib Versus Those Receiving Tumor Necrosis Factor Inhibitors : An Observational Cohort Study. Arthritis Rheumatol., 2019, 71(6) : 892-900.

19. SANDBORN WJ, PANÉS J, SANDS BE, REINISCH W, SU C, LAWENDY $\mathrm{N}$, et al. Venous thromboembolic events in the tofacitinib ulcerative colitis clinical development programme. Aliment. Pharmacol. Ther., 2019, 50(10) : 1068-76.

20. DEEPAK P, ALAYO QA, KHATIWADA A, LIN B, FENSTER M, DIMOPOULOS C, et al. Safety of Tofacitinib in a Real-World Cohort of Patients With Ulcerative Colitis. Clin. Gastroenterol. Hepatol., 2020.

21. US FDA. FDA approves Boxed Warning about increased risk of blood clots and death with higher dose of arthritis and ulcerative colitis medicine tofacitinib (Xeljanz, Xeljanz XR): FDA drug safety communication. Washington, DC July 26, 2019. Available from : https://www.fda.gov/drugs/ drug-safety-and-availability/fda-approves-boxed-warning-about-increasedrisk-blood-clots-and-death-higher-dose-arthritis-and.

22. Magro F, Soares JB, Fernandes D. Venous thrombosis and prothrombotic factors in inflammatory bowel disease. World J Gastroenterol. 2014, 20(17) : 4857-72.

23. KOUTROUBAKIS IE, SFIRIDAKI A, TSIOLAKIDOU G, COUCOUTSI C, THEODOROPOULOU A, KOUROUMALIS EA. Plasma thrombinactivatable fibrinolysis inhibitor and plasminogen activator inhibitor-1 levels in inflammatory bowel disease. Eur. J. Gastroenterol. Hepatol., 2008, 20(9) : 912-6.

24. BOLLEN L, VANDE CASTEELE N, PEETERS M, VAN ASSCHE G, FERRANTE M, VAN MOERKERCKE W, et al. The Occurrence of Thrombosis in Inflammatory Bowel Disease Is Reflected in the Clot Lysis Profile. Inflamm. Bowel Dis., 2015, 21(11) : 2540-8.

25. BOLLEN L, VANDE CASTEELE N, PEETERS M, BESSONOV K, VAN STEEN K, RUTGEERTS P, et al. Short-term effect of infliximab is reflected in the clot lysis profile of patients with inflammatory bowel disease: a prospective study. Inflamm. Bowel Dis., 2015, 21(3) : 570-8.

26. KOUTROUMPAKIS EI, TSIOLAKIDOU G, KOUTROUBAKIS IE. Risk of venous thromboembolism in patients with inflammatory bowel disease. Semin. Thromb. Hemost., 2013, 39(5) : 461-8.

27. NGUYEN GC, SAM J. Rising prevalence of venous thromboembolism and its impact on mortality among hospitalized inflammatory bowel disease patients. Am. J. Gastroenterol., 2008, 103(9) : 2272-80. 
28. HARBORD M, ANNESE V, VAVRICKA SR, ALLEZ M, BARREIRODE ACOSTA M, BOBERG KM, et al. The First European Evidence-based Consensus on Extra-intestinal Manifestations in Inflammatory Bowel Disease. J. Crohns Colitis, 2016, 10(3) : 239-54.

29. NGUYEN GC, BERNSTEIN CN, BITTON A, CHAN AK, GRIFFITHS AM, LEONTIADIS GI, et al. Consensus statements on the risk, prevention, and treatment of venous thromboembolism in inflammatory bowel disease : Canadian Association of Gastroenterology. Gastroenterology, 2014, 146(3) : 835-48.e6.

30. PAPA A, PAPA V, MARZO M, SCALDAFERRI F, SOFO L, RAPACCINI GL, et al. Prevention and treatment of venous thromboembolism in patients with IBD : a trail still climbing. Inflamm. Bowel Dis., 2015, 21(5) : 1204-13.

31. RA G, THANABALAN R, RATNESWARAN S, NGUYEN GC. Predictor and safety of venous thromboembolism prophylaxis among hospitalized inflammatory bowel disease patients. J. Crohns Colitis, 2013, 7(10) : e47985 .

32. CURTIS KM, TEPPER NK, JATLAOUI TC, BERRY-BIBEE E, HORTON LG, ZAPATA LB, et al. U.S. Medical Eligibility Criteria for Contraceptive Use, 2016. MMWR Recomm. Rep., 2016, 65(3) : 1-103.

33. PELLINO G, SCIAUDONE G, CAPRIO F, CANDILIO G, DE FATICO GS, REGINELLI A, et al. Hormonal contraceptives and venous thromboembolism: Are inflammatory bowel disease patients at increased risk? A retrospective study on a prospective database. Ann. Med. Surg. (Lond), 2015, 4(4) : 462-6.

34. LOBO JL, GARCIA-FUERTES JA, TRUJILLO-SANTOS J, MERAH A, BLANCO-MOLINA MA, CASADO I, et al. Anticoagulant therapy for venous thromboembolism in patients with inflammatory bowel disease. Eur J. Gastroenterol. Hepatol., 2018, 30(5) : 526-30.

35. ALOTAIBI G, ALSALEH K, WU C, MCMURTRY MS. Dabigatran, rivaroxaban and apixaban for extended venous thromboembolism treatment : network meta-analysis. Int. Angiol., 2014, 33(4) : 301-8.

36. IORIO A, KEARON C, FILIPPUCCI E, MARCUCCI M, MACURA A, PENGO V, et al. Risk of recurrence after a first episode of symptomatic venous thromboembolism provoked by a transient risk factor : a systematic review. Arch. Intern. Med., 2010, 170(19) : 1710-6.

37. ALKIM H, KOKSAL AR, BOGA S, SEN I, ALKIM C. Etiopathogenesis, Prevention, and Treatment of Thromboembolism in Inflammatory Bowel Disease. Clin. Appl. Thromb. Hemost., 2017, 23(6) : 501-10.

38. MARSHALL JK, OTLEY AR, AFIF W, BERNSTEIN CN, HOOKEY L, LEONTIADIS G, et al. Canadian Association of Gastroenterology position statement regarding the use of thiopurines for the treatment of inflammatory bowel disease. Can. J. Gastroenterol. Hepatol., 2014, 28(7) : 371-2. 